\title{
La importancia de incorporar la hidrodinámica de la interfaz salina en la gestión de los recursos minerales y ecosistemas de los salares
}

\author{
Miguel Ángel Marazuela ${ }^{(1,2,3)}$, Enric Vázquez Suñé(1), Emilio Custodio(2,4), Carlos Ayora(1), Teresa \\ Palma $^{(1)}$ y Alejandro García-Gil ${ }^{(5)}$ \\ (1) Instituto de Diagnóstico Ambiental y Estudios del Agua, CSIC, C/ Jordi Girona 18-26, 08034 Barcelona, España. \\ (2) Departamento de Ingeniería Civil y Ambiental, Universidad Politécnica de Cataluña (UPC), C/ Jordi Girona 1-3, 08034, \\ Barcelona, España. \\ (3) University of Vienna, Centre for Microbiology and Environmental Systems Science, Environmental Geosciences, \\ Vienna, Austria. \\ (4) Real Academia de Ciencias Exactas, Físicas y Naturales de España. \\ (5) Instituto Geológico y Minero de España (IGME), Manuel Lasala 44, 9 B, 50006 Zaragoza, España. \\ mamarazuela@outlook.com
}

\begin{abstract}
RESUMEN
Los salares son una fuente importante de minerales y especialmente de litio. Además, lagunas y humedales de alto valor ecológico están asociados frecuentemente a la zona de la interfaz salina (zona de mezcla) resultante del contraste de densidades entre el agua dulce recargada en el frente montañoso y la salmuera evaporada desde el salar que se ha instalado en la depresión. Esta zona se caracteriza por la presencia de flujos verticales, que condicionan la hidrodinámica a escala de cuenca. Es necesario establecer una metodología que permita incorporar el efecto de la interfaz salina en los modelos de agua subterránea que están enfocados a la gestión de sus recursos minerales y valores ecológicos. Dicha metodología consta de 3 fases: 1) recogida de datos físico-químicos en campo, 2) cartografía 3D de la zona de mezcla y 3) modelación numérica. Se presenta una cartografía tridimensional (3D) de la zona de mezcla del Salar de Atacama. Las diferentes geometrías de la zona de mezcla se han interpretado utilizando un modelo numérico de flujo y transporte de solutos. El resultado del modelo muestra una pendiente de la zona de mezcla similar a la obtenida a través de los datos de campo, la cual es más laxa que en los modelos previos, que consideraron un medio poroso homogéneo. Cuanto mayor es la permeabilidad del acuífero superior, menores son los niveles hidráulicos $y$, por lo tanto, menor es la pendiente de la interfaz salina. La presencia de unos pocos metros de materiales altamente permeables (abanicos aluviales o evaporitas karstificadas) en las unidades estratigráficas superiores de los salares es suficiente para modificar la geometría regional de la zona de mezcla.

Se propone utilizar esta cartografía 3D como base para corregir los niveles piezométricos de agua dulce y de agua mezcla a niveles equivalentes de salmuera, con el fin de reducir el coste computacional de futuros modelos numéricos tridimensionales enfocados a la gestión de las aguas subterráneas de los salares.
\end{abstract}

Palabras clave: agua subterránea, litio, intrusión salina, salar, salmuera.

\section{The importance of considering the hydrodynamics of the saline interface in the managements of mineral resources and ecosystems of salt flats}

\begin{abstract}
Salt flats are an important source of minerals and especially of lithium. In addition, lakes and wetlands of high ecological value are frequently associated with the saline interface zone (mixing zone) resulting from the density contrast between the freshwater coming from the rainfal infiltration on the mountains and the brine produced mainly by evaporation in the depressions. This zone is characterized by vertical flows that condition
\end{abstract}


the hydrodynamics at a basin scale. It is necessary to establish a methodology that allows the incorporation of the effect of the saline interface into groundwater models for the management of the mineral resources and ecological values. This methodology consists of 3 steps: 1) physical-chemical data collection in the field, 2) 3D mapping of the mixing zone and 3) numerical modelling.

A three-dimensional (3D) mapping of the mixing zone of the Salar de Atacama is presented. The mixing zone geometry was interpreted using a numerical flow and solute transport model. The results from the model show a slope of the mixing zone that is similar to that obtained with the field data, which is more relaxed than in the previous models that considered a homogeneous porous medium. The higher the permeability of the upper aquifer is, the lower the slope and the shallower the mixing zone become. The presence of a few metres of highly permeable materials (alluvial fans or karstified evaporites) in the upper stratigraphic units of the salt flats is enough to condition the regional geometry of the saline interface.

The use of this 3D mapping is proposed as a basis for the correction of the freshwater and mixing water heads to brine heads to reduce the computational cost of $3 D$ numerical models for groundwater management in salt flats.

Keywords: brine, lithium, groundwater, salt flat, saltwater intrusion.

\section{Introducción}

Los salares son sistemas endorreicos e hidrogeológicamente cerrados, asociados frecuentemente con climas áridos o hiperáridos y, por lo tanto, con tasas de evaporación muy altas. Esto hace que el agua superficial se evapore rápidamente, favoreciendo que la superficie freática se sitúe justo por debajo del nivel del terreno (Muñoz-Pardo et al., 2004; Marazuela et al., 2019ab). Las altas tasas de evaporación, procesos de disolución y remobilización de minerales e hidrotermalismo favorecen el aumento de la concentración de algunos elementos químicos (litio, potasio, boro, etc.) en las salmueras residuales subterráneas, que son muy apreciados comercialmente y demandados por la industria de la automoción y farmacéutica (Marazuela et al., 2020b). Como estas materias primas se encuentran disueltas en las salmueras subterráneas, su explotación resulta sencilla y con un bajo coste económico, ya que basta bombearla mediante pozos y posteriormente evaporarla fraccionadamente en pozas hasta que precipita el mineral de interés.

Sin embargo, estos sistemas hidrogeológicos no solo son interesantes desde un punto de vista económico sino que también albergan ecosistemas únicos en el mundo. Los principales ecosistemas suelen localizarse en las lagunas y humedales de la zona marginal de los salares, donde la superficie freática alcanza localmente la superficie del terreno (DGA 1977; Ortiz et al., 2014; Salas et al., 2010; Marazuela et al., 2018). Debido a la fragilidad y especialización ecológica de estos ecosistemas, los bombeos llevados a cabo por la industria minera suponen una amenaza potencial para su preservación (Marazuela et al., 2020a). Por un lado, una disminución del nivel freático puede secar las lagunas y humedales. Por otro lado, un avance de la interfaz agua dulce-salmuera hacia el centro del salar puede modificar la salinidad de sus aguas. Es por esto que resulta de gran importancia conocer la hidrodinámica y funcionamiento de los salares y, en concreto, de su zona marginal donde aflora la interfaz salina.
El modelo hidrogeológico más aceptado asume que la recarga principal de la cuenca se produce en las montañas vecinas a la depresión donde se ubica el salar, donde se alcanzan las cotas más elevadas y las precipitaciones son mayores (Marazuela et al., 2019a). Por el contrario, la salida de agua del sistema cerrado se produce por evaporación freática desde el salar. Desde un punto de vista hidrodinámico, se asume que las salmueras densas producidas por la evaporación se hunden en el salar, formando parte de celdas de convección, cuyos ramales ascendentes se dirigen hacia la superficie (flujos verticales) en los márgenes del salar, donde las salmueras se mezclan con el agua subterránea dulce o poco salina que fluye desde el frente montañoso (Marazuela et al., 2018). La zona de contacto entre estos dos fluidos miscibles, pero de diferentes densidades, es la interfaz salina o zona de mezcla. Aunque la modelación de la interfaz salina ha sido abordada en trabajos previos (Tejeda et al., 2003; Vásquez et al., 2013), la morfología y pendiente de las interfaces resultantes de dichos modelos no mostraba los mismos resultados. Marazuela et al., (2018) explican dichas diferencias por la influencia de la heterogeneidad vertical de estos sistemas y propone su incorporación en modelos hidrogeológicos 3D de bajo coste computacional mediante correcciones de densidad de los niveles piezométricos Un claro ejemplo de la necesidad de conocer en profundidad la geometría y funcionamiento de la interfaz salina y de su incorporación a los modelos hidrogeológicos 3D es el modelo numérico elaborado por GeoHidrología Consultores Ltda. (2013). En este trabajo se evidencia la dificultad de reproducir los flujos verticales asociados a la zona marginal del Salar de Atacama así como de reproducir el efecto "barrera" que la zona de mezcla ejerce entre el flujo de agua dulce proveniente de las montañas y el núcleo del salar. La única forma con la que consiguen reproducir los flujos verticales es incluyendo una barrera vertical impermeable en la zona de mezcla, la cual no representa a ninguna característica geológica sino que simplemente es un artefacto numérico. 
El objetivo de este trabajo es mostrar los últimos avances científicos realizados en la caracterización geométrica e hidrodinámica de la interfaz salina del Salar de Atacama, para extraer una metodología que permita la gestión sostenible de sus ecosistemas y de sus recursos minerales mediante la modelización numérica 3D del flujo subterráneo y que sea extrapolable a otros salares.

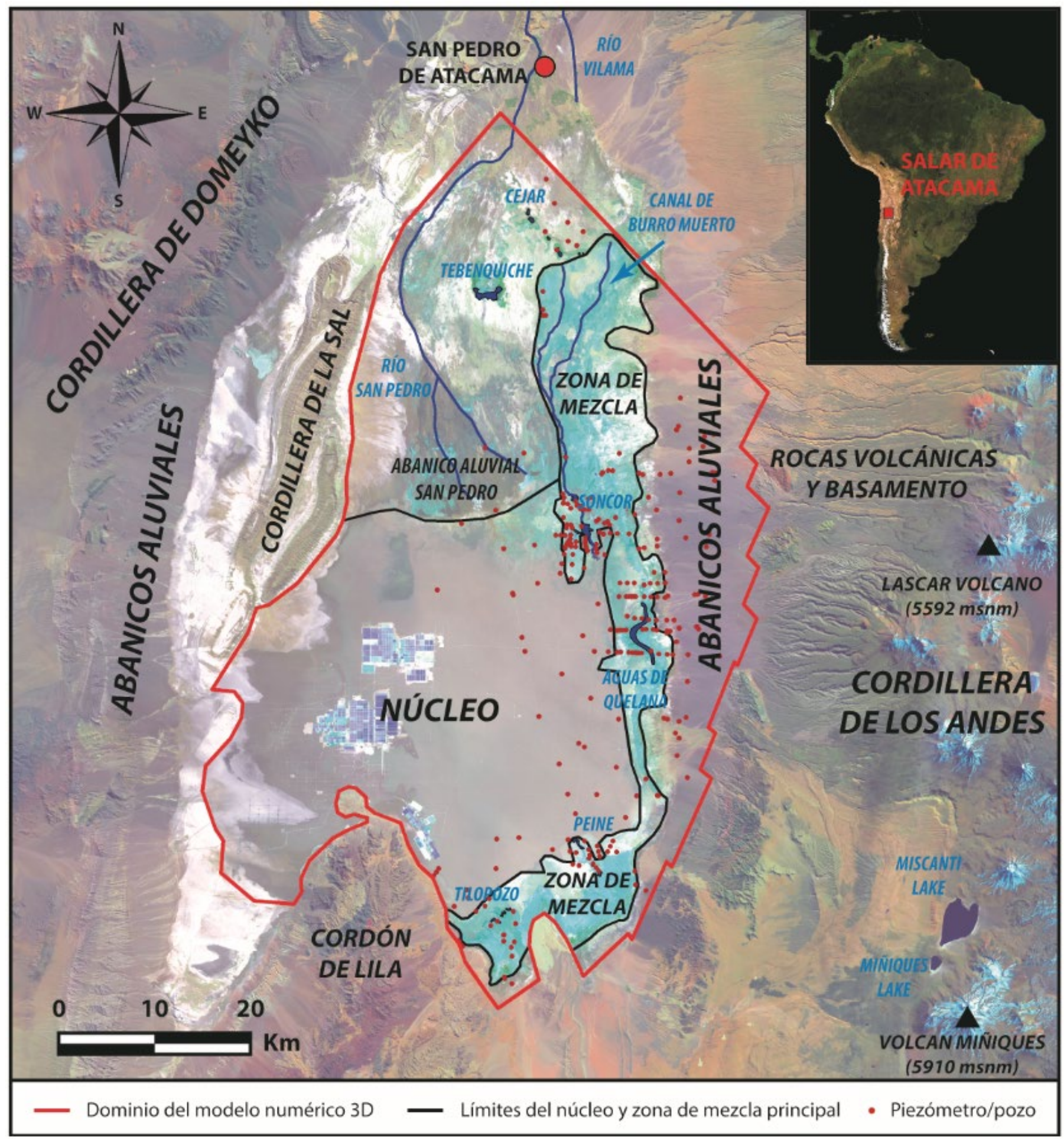

Figura 1. Localización geográfica de la zona de estudio.

Figure 1. Geographical location of the study area. 


\section{Situación geográfica y caracterización hidrogeológica}

El Salar de Atacama se localiza en el Norte de Chile, entre $23^{\circ}$ y $24^{\circ}$ de latitud Sur y $68^{\circ}$ y $69^{\circ}$ de longitud Oeste (Fig. 1). Está ubicado en la II Región, Antofagasta, dentro de los límites del municipio de San Pedro de Atacama. La cuenca hidrográfica donde se ubica el salar tiene forma ovalada, con el eje longitudinal en dirección N-S y una extensión de aproximadamente $20,000 \mathrm{~km}^{2}$. Limita al Oeste con la Cordillera de la Sal y la Cordillera de Domeyko, al Este con la Cordillera de los Andes, cuyos picos más altos corresponden al arco volcánico actual a $>5500 \mathrm{~m} \mathrm{snm}$ (metros sobre el nivel del mar), al Norte con la unión de la Cordillera Domeyko y la Cordillera de los Andes y al Sur con el Cordón de Lila.

Debido a que la Cordillera de los Andes actúa como una barrera geográfica al movimiento atmosférico, el Salar de Atacama se caracteriza por un clima hiperárido. En promedio, el salar recibe menos de $20 \mathrm{~mm} / a n ̃ o$ de precipitación y en las montañas la precipitación media alcanza $160 \mathrm{~mm} / a$ ño (Marazuela et al., 2019a). El principal aporte de agua al salar es subterráneo y se recarga por la lluvia y la fusión de nieve en la CordiIlera de los Andes. Esta agua se desplaza por diferencia de gradientes piezométricos desde las montañas hasta la zona marginal o zona de mezcla del Salar de Atacama, pasando por los abanicos aluviales que ha- cen de contacto entre rocas volcánicas y el salar.

En el margen oriental del Salar de Atacama y más concretamente en la superficie de la zona de mezcla, hay varias lagunas y humedales, en algunos casos declarados sitios Ramsar, como es el caso del Sistema Lagunar Soncor y Aguas de Quelana. Esta zona se encuentra constituida por carbonatos y yesos, que dan paso a los depósitos de halita del núcleo del salar siguiendo la secuencia de precipitación típica de la evaporación del agua. La Formación Vilama que constituye el núcleo del Salar alcanza los $1200 \mathrm{~m}$ de espesor (Jordan et al., 2007).

La zona superficial es la que presenta una hidrodinámica más activa por su mayor permeabilidad, diferenciándose dos acuíferos, A y B, dominados por halita, que se encuentran separados por un acuitardo $A B$ constituido por yesos, carbonatos y depósitos detríticos finos (Marazuela et al., 2019a). EI espesor de estas tres unidades hidroestratigráficas está fuertemente afectado por las fallas Salar y Tucúcaro. El acuífero A tiene un espesor de alrededor de $20 \mathrm{~m}$ en el bloque occidental (bloque levantado) y de $40 \mathrm{~m}$ en el bloque oriental (bloque hundido). El acuitardo $A B$ tiene un espesor inferior a $1 \mathrm{~m}$ en el bloque occidental y de más de 10 metros en el bloque oriental. El acuífero $B$ pasa de unos $40 \mathrm{~m}$ en el bloque levantado a alcanzar más de $400 \mathrm{~m}$ en el bloque hundido.

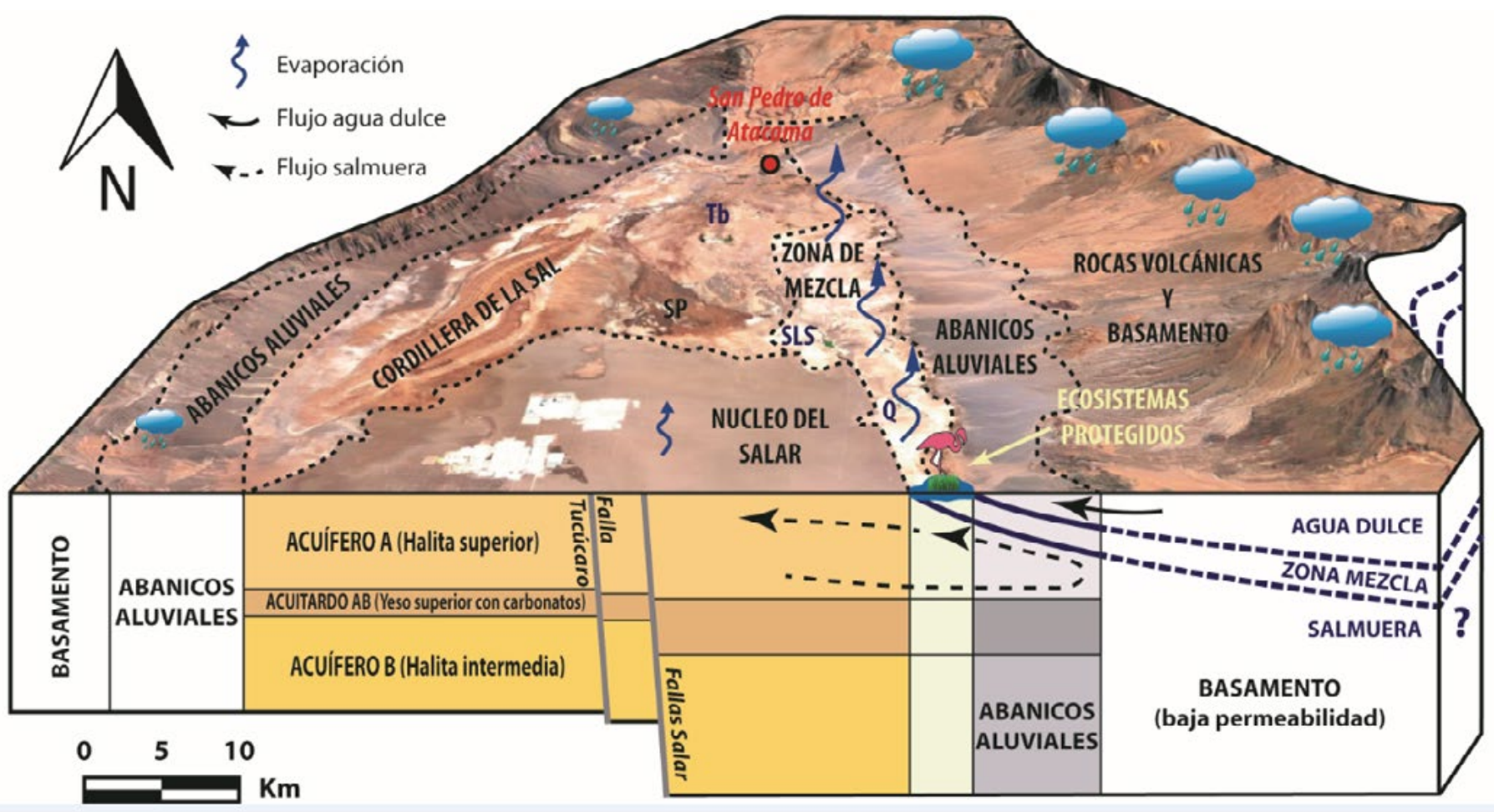

Figura 2. Esquema conceptual del funcionamiento hidrogeológico del Salar de Atacama (Modificado de Marazuela et al., 2019a).Tb, laguna Tebenquiche; SLS, sistema laguna Soncor; Q, sistema lagunar Aguas de Quelana; SP, abanico aluvial San Pedro.

Figure 2. Conceptual scheme of the hydrogeological functioning of the Salar de Atacama (Modified from Marazuela et al., 2019a). Th, Tebenquiche lagoon ; SLS, Soncor lagoon system; Q, Aguas de Quelana lagoon system; SP, San Pedro alluvial fan. 


\section{Metodología}

En el presente trabajo se propone una metodología completa para la modelación numérica del flujo subterráneo que permita la gestión sostenible de los recursos minerales y ecosistemas de los salares, aplicándola al caso de estudio del Salar de Atacama. La metodología consta de tres fases: 1) muestreo de las aguas subterráneas y medición de parámetros físico-químicos, 2) cartografía 3D de la interfaz salina y 3) modelación numérica de la interfaz salina a escala local y elaboración de modelos numéricos 3D que sean eficientes computacionalmente.

En una red de monitoreo de 310 puntos de observación (pozos, piezómetros, zanjas y cursos de aguas superficiales), se midió la profundidad y columna de agua, la conductividad eléctrica (CE), la densidad y los sólidos totales disueltos. Para aumentar el número de datos disponibles para la cartografía tridimensional de la zona de mezcla, se han obtenido correlaciones entre densidad, conductividad eléctrica y sólidos totales disueltos, que pueden consultarse en Marazuela et al., 2018. Los niveles piezométricos y los parámetros físico-químicos se midieron en el campo. Los mismos parámetros físico-químicos y las concentraciones de solutos se midieron también en el laboratorio siguiendo procedimientos estándar. Además, en algunos pozos se realizaron perfiles verticales de conductividad eléctrica (CE). La mayoría de los datos utilizados para este proyecto de investigación fueron proporcionados por la compañía SOM (Sociedad Química y Minera de Chile), con algunos registros que datan del año 1984,

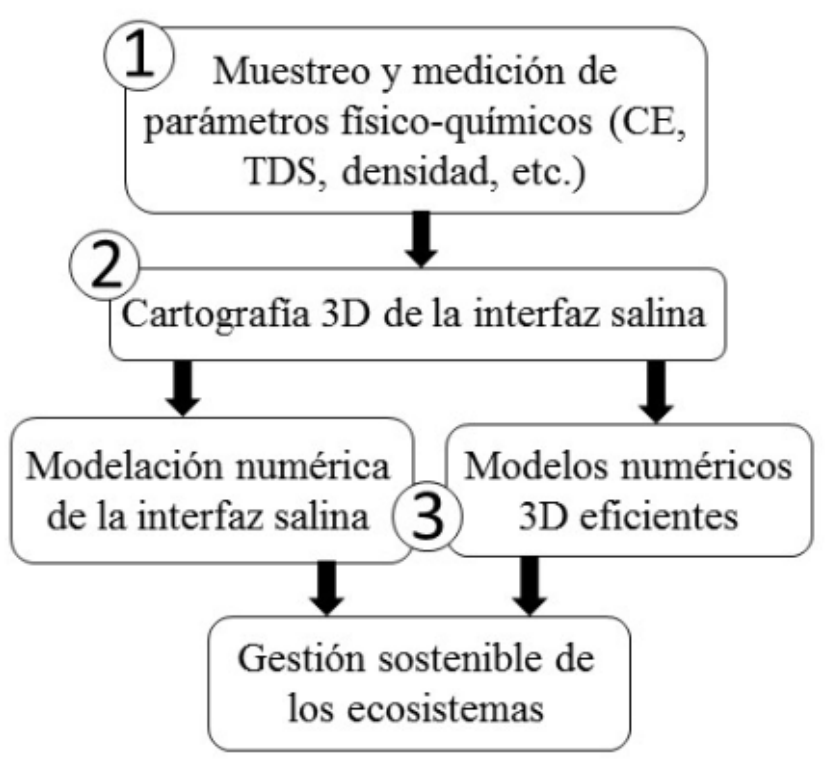

Figura 3. Propuesta metodológica para la caracterización, modelación y gestión de los salares y sus ecosistemas.

Figure 3. Proposed methodology to characterize, model and manage the salt flats and their ecosystems. lo que permite conocer la evolución temporal.

El análisis de imágenes satelitales LANDSAT 8 se ha utilizado para cartografiar la zona de mezcla en superficie e identificar sus distintas zonas (interna, intermedia y externa). Se ha correlacionado la información extraída del análisis visual de las imágenes satelitales con los datos físico-químicos recogidos en superficie y en profundidad (pozos), para poder cartografiar en profundidad la zona de mezcla.

Se ha modelado numéricamente en 2D una sección vertical sintética de la zona de mezcla del Salar de Atacama, utilizando el código FEFLOW, el cual resuelve las ecuaciones acopladas de flujo y transporte de solutos teniendo en cuenta las variaciones de densidad. Además, se ha justificado la necesidad de corregir los niveles por contrastes de densidad en los modelos numéricos 3D aplicados a la gestión de los recursos mineros y los ecosistemas de los salares.

\section{Resultados y discusión}

\section{Cartografía de la zona de mezcla}

La zona de mezcla entre la salmuera y el agua dulce de recarga se evidencia en la superficie como una franja de color blanquecino de entre 2 y $10 \mathrm{~km}$ de ancho en el margen oriental y suroriental del salar y de hasta $40 \mathrm{~km}$ hacia el norte del mismo (Figs. 1, 2 y 4). La zona de mezcla del Salar de Atacama se extiende a lo largo de los márgenes noreste, este y sureste del salar. Las densidades superficiales más cercanas a $1,23 \mathrm{~kg} / \mathrm{L}$ (salmuera saturada de halita) se midieron en el núcleo, mientras que las densidades más cercanas a $1,00 \mathrm{~kg} / \mathrm{L}$ se midieron en la zona de mezcla externa y en la zona de recarga.

Dentro de la zona de mezcla, su parte central o zona de mezcla intermedia (MMZ) se identificó mediante el uso de imágenes de satélite (Fig. 4) ya que coincide frecuentemente con humedales y pequeñas lagunas perennes o estacionales, fácilmente reconocibles. Esto sirvió para diferenciar otros dos dominios hidráulicos: la zona de mezcla interna (IMZ) y la zona de mezcla externa (EMZ).

La cartografía tridimensional de la zona de mezcla se realizó identificando la posición de su superficie central $\left(E C=150 \mathrm{mS} \cdot \mathrm{cm}^{-1}\right)$, superior $(E C=100$ $\mathrm{mS} \cdot \mathrm{cm}^{-1}$ ) e inferior $\left(E C=200 \mathrm{mS} \cdot \mathrm{cm}^{-1}\right.$ ) (Fig. 5), utilizando las mediciones de los parámetros físico-químicos realizadas en los pozos. Estas superficies se utilizan para delinear la zona de mezcla principal, que es la región en la que el gradiente de salinidad cambia más rápidamente. En general, la pendiente presenta una geometría mucho más laxa y menos verticalizada que la resultante de los modelos previos de Tejeda et al. (2003) y Vásquez et al. (2013).

En el sector oriental y suroriental del salar (ver secciones $\mathrm{B}$ y $\mathrm{C}$ de la Fig. 5), la zona de mezcla muestra generalmente una pendiente moderada entre el núcleo del salar y las lagunas, es decir en la IMZ. En la 
zona de las lagunas y en la EMZ la pendiente se acentúa, lo cual favorece el ascenso rápido del agua subterránea que alimenta las lagunas (Fig. 5).

En cambio, en el sector norte, la interfaz salina muestra en toda su extensión una pendiente mucho menor en que los sectores oriental y suroriental (ver sección A de la Fig. 5). Esto es coherente con la geometría ovalada de la cuenca y sobretodo con la distribución de la recarga por lluvia producida a lo largo de la misma.

Como la principal recarga por lluvia se produce en las subcuencas del sector E y SW del salar (Marazuela et al., 2019a), existe un desplazamiento de la zona de mezcla hacia el salar mucho mayor que en el sector norte, donde hay una menor recarga y los niveles piezométricos de agua dulce son menores, produciendo un menor desplazamiento de la interfaz salina y por lo tanto un avance mayor de la intrusión de salmuera. En este sector, a diferencia de la zona E y SW, el ascenso de agua subterránea profunda hasta la superficie no solo coincide con la MMZ, sino que en la IMZ también están presentes vertientes (manantiales) de carácter puntual (Fig. 5B). Estas vertientes se en-
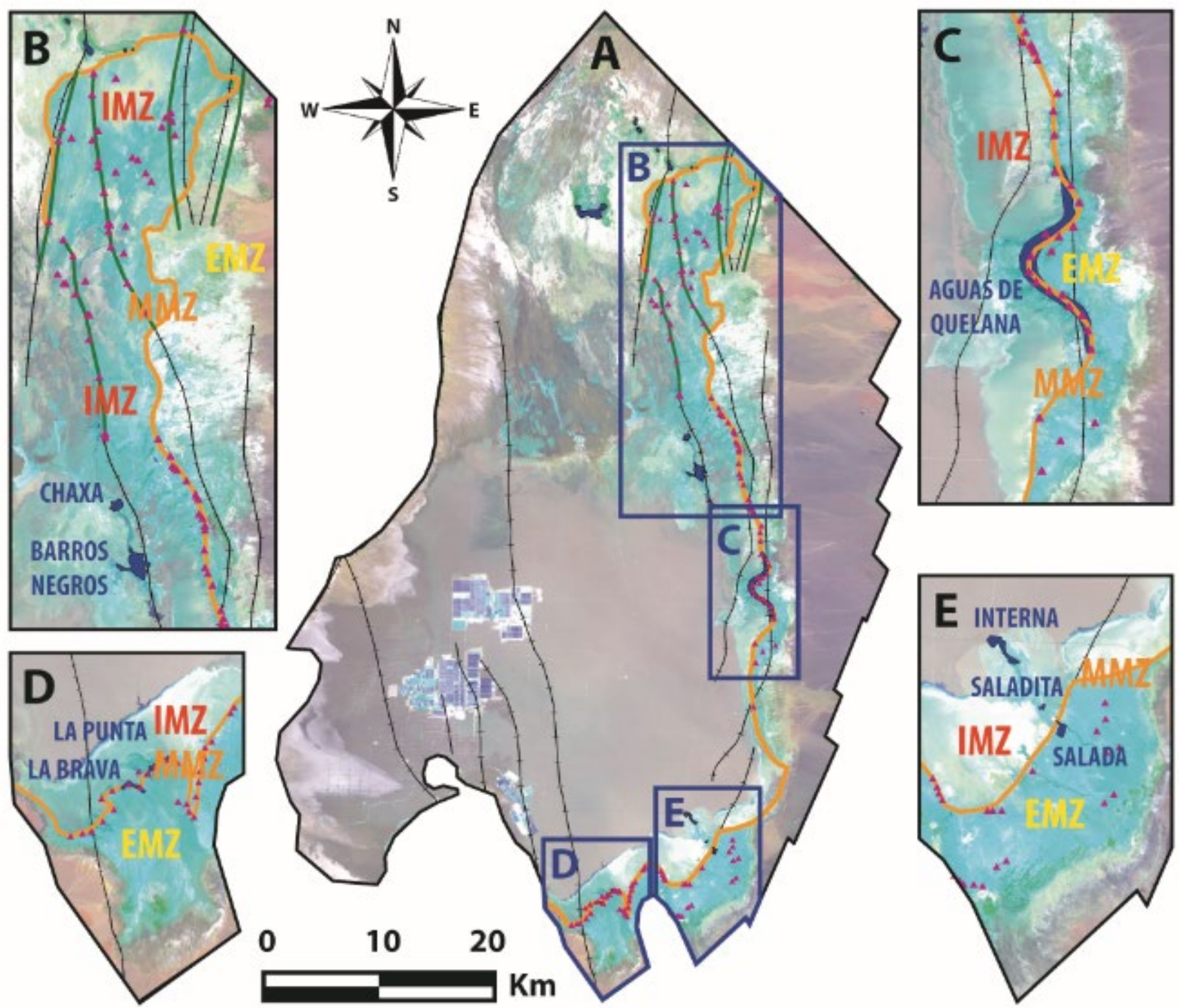

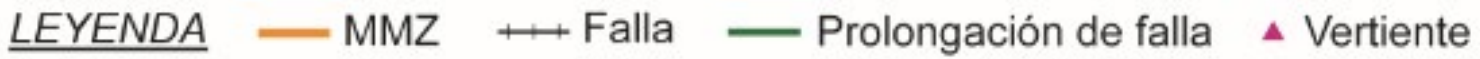

Figura 4. Cartografía superficial de la zona de mezcla del Salar de Atacama (modificado de Marazuela et al., 2018).

Figure 4. Mapping of the mixing zone surface of the Salar de Atacama (modified from Marazuela et al., 2018). 
cuentran alineadas siguiendo los planos estructurales identificados por la Universidad de Chile (2016) o su prolongación, lo que parece indicar que son el resultado de ascensos de agua subterránea concentrados en los planos de falla.

\section{Modelación numérica de la interfaz salina}

Para comprender la geometría de la zona de mezcla de los salares y poder describir la geometría descrita en el apartado anterior, se ha realizado el modelo numérico 2D de un perfil vertical idealizado, perpendicular al borde. El dominio modelado tiene 25000 $\mathrm{m}$ de largo y $1200 \mathrm{~m}$ de ancho (Fig. 6). La longitud seleccionada incluye $10000 \mathrm{~m}$ de núcleo, $5000 \mathrm{~m}$ de zona de mezcla y $10000 \mathrm{~m}$ de abanicos aluviales. El espesor corresponde a la potencia de la Formación Vilama. El límite derecho (oriental) es el contacto entre las rocas del basamento o las rocas volcánicas, que son consideradas impermeables a efectos prácticos, $y$ los abanicos aluviales. El límite izquierdo (occidental) se ubica dentro del núcleo del salar, lo suficientemente alejado de la zona de mezcla para no condicionar los resultados. El límite inferior representa la base del acuífero B. El límite superior se asume como el nivel freático. La malla del modelo está constituida por
153000 elementos triangulares.

Se han considerado tres capas simplificadas que representan la secuencia regional acuífero-acuitardo-acuífero, que regionalmente se identifica tanto en el núcleo como en los abanicos aluviales, aunque desde el punto de vista litológico y geo-cronológico no siempre exista una relación clara (Fig. 2).

Los límites izquierdo, inferior y derecho se tratan como sin flujo para el agua y la masa. En la parte superior se seleccionaron diferentes condiciones de contorno para representar los procesos de evaporación y recarga. La lluvia en la sección de la cuenca considerada varía entre 20 y $140 \mathrm{~mm} / a n ̃ o$, de la que el $25 \%$ se evapora. Estos valores fueron adaptados a la longitud prescrita (Marazuela et al., 2018). La recarga se aplicó en la parte superior, para no condicionar la geometría de la zona de mezcla en el límite derecho. Se fijó un nivel de agua (condición de Dirichlet) en el núcleo de halita y en la zona de mezcla interna. EI valor de la recarga y por lo tanto del aporte lateral de agua es un valor incierto y deberá ser objeto de estudios futuros de detalle. El alto valor adoptado aunque puede parecer algo elevado, se encuentra dentro de los valores posibles si se cuenta el papel importante de la fusión de nieve en esta área andina, según los estudios en curso por la Universidad Católica de
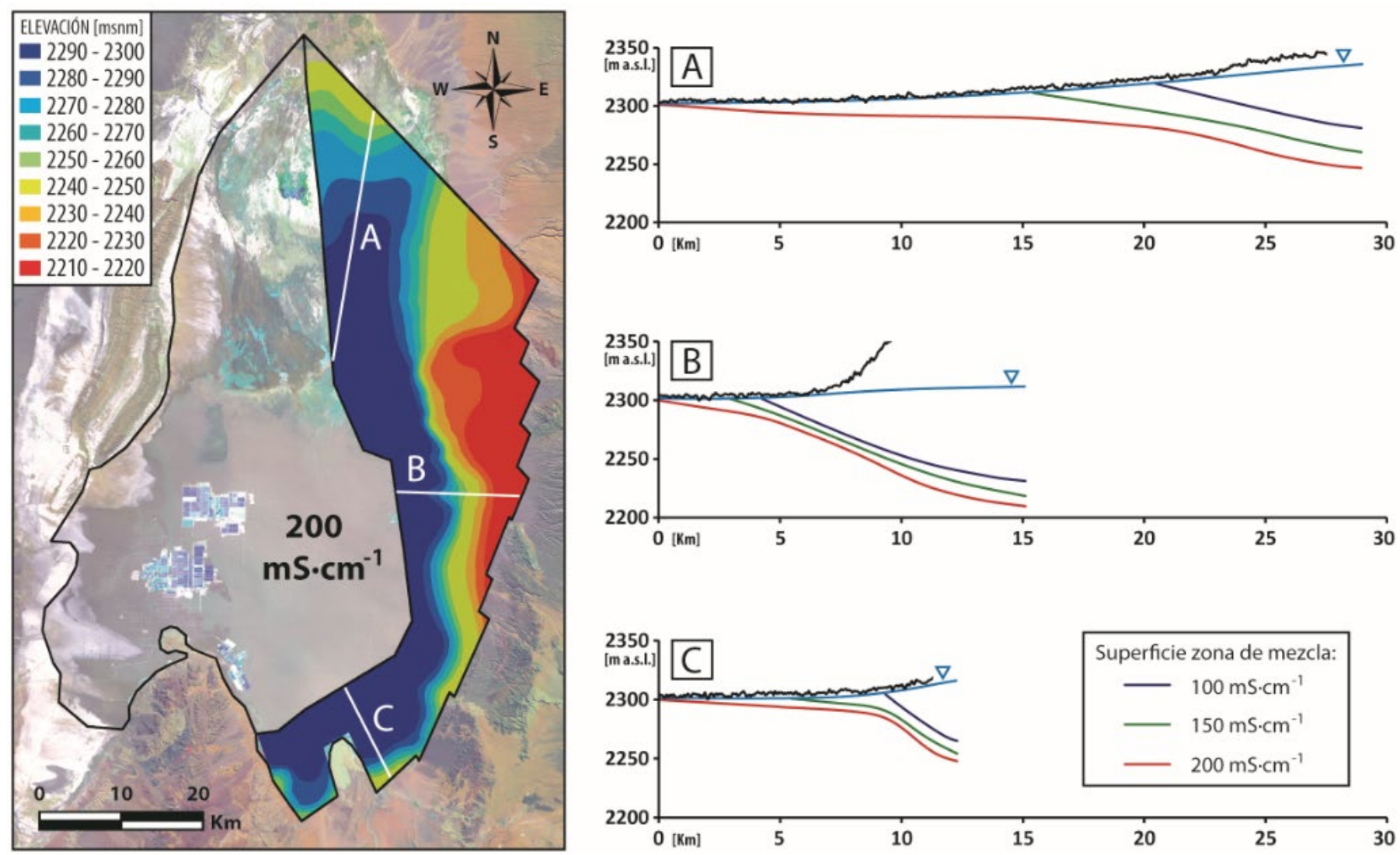

Figura 5. Cartografía 3D de la zona de mezcla del Salar de Atacama.

Figure 5. 3D mapping of the mixing zone of the Salar de Atacama. 
Antofagasta (Urrutia et al, 2019; Herrera et al., 2016; Acosta y Custodio, 2008), y la experiencia sobre las condiciones de recarga en las áreas áridas del Norte de Chile (Herrera y Custodio, 2014a; Herrera et al., 2018; Gamboa et al., 2019) y de las Islas Canarias (Custodio, 1992; Custodio y Custodio-Ayala, 2001; Herrera y Custodio, 2014b).y el efecto de épocas pasadas más lluviosas (Custodio et al., 2018).

Como se comenta más adelante, la incertidumbre de la recarga afecta solo moderadamente a la posición de la zona de mezcla.

Para el transporte de masa se normalizaron concentraciones fijas extremas de solutos de $1,23 \mathrm{~kg} / \mathrm{L}$ para el núcleo y $1,00 \mathrm{~kg} / \mathrm{L}$ para la zona de recarga por lluvia.

Se llevó a cabo una simulación estacionaria para obtener los niveles piezométricos iniciales. Para la condición de masa inicial se asignó una concentración de $1,00 \mathrm{~kg} / \mathrm{L}$, que corresponde a un escenario hipotético

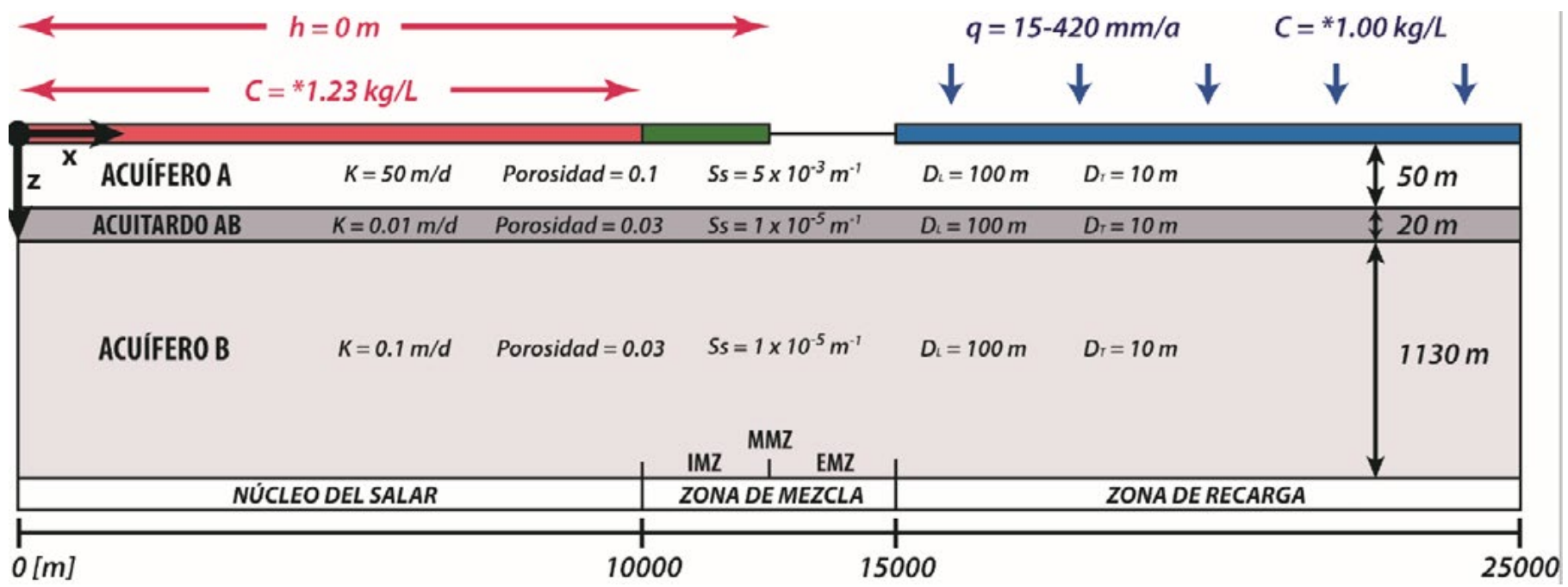

Figura 6. Geometría y condiciones de contorno del modelo numérico 2D. $\mathrm{K}=$ conductividad hidráulica, Ss = coeficiente de almacenamiento específico, $\mathrm{h}=$ nivel piezométrico, $\mathrm{q}=$ flujo de agua: $\mathrm{DL}=$ dispersividad longitudinal y $\mathrm{DT} \mathrm{s}=$ dispersividad transversal.

Figure 6. Geometry and boundary conditions of the $2 D$ numerical model. $K=$ hydraulic conductivity, Ss = the specific storage coefficient, $h=$ water head, $D L=$ longitudinal dispersivity and $D T=$ transversal dispersivity.
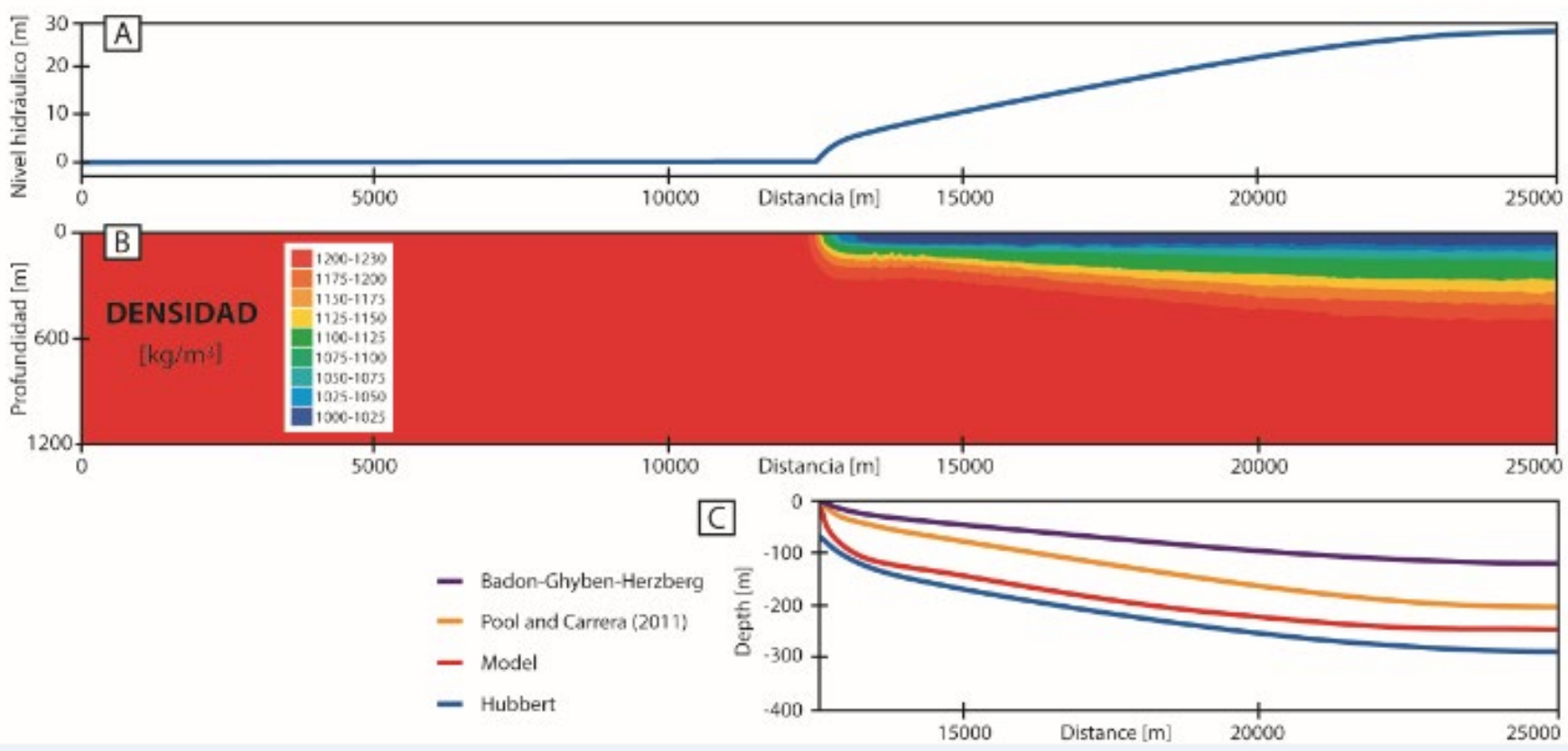

Figura 7. Resultado del modelo numérico de la interfaz salina del Salar de Atacama (Modificado de Marazuela et al., 2018).

Figure 7. Results of the numerical model of the Salar de Atacama saline interface (Modified from Marazuela et al., 2018). 
en el tiempo 0 donde todas las aguas subterráneas son dulces. El modelo se corrió durante varios miles de años, con control de paso de tiempo semiautomático, para alcanzar un régimen cuasi-estacionario. Los parámetros hidráulicos son los deducidos de ensayos de bombeo y tomados de referencias bibliográficas (IDAEA-CSIC, 2017).

Los resultados se muestran en la Fig. 7. La geometría de la zona de mezcla presenta una pendiente muy baja, que le permite penetrar por completo bajo los abanicos aluviales. Este tipo de zona de mezcla de muy baja pendiente corresponde a zonas muy áridas y de alta permeabilidad, de forma análoga al caso 4 descrito por Duffy y Al-Hassan (1988).

En estado cuasi-estacionario, los efectos de la densidad han permitido diferenciar cinco sectores con comportamientos hidráulicos muy diferentes: núcleo, zona de mezcla interna (IMZ), zona de mezcla intermedia (IMZ), zona de mezcla externa (EMZ) y zona de recarga. La hidrodinámica en el núcleo está controlada por celdas de convección en continua evolución. Estas celdas de convección, cuyo motor es la evapo- ración desde el núcleo y la zona de mezcla superficial, se hacen cada vez más densas, colapsan, se hunden y provocan el movimiento ascendente de la salmuera menos densa. La presencia de capas de baja permeabilidad, como el acuitardo $A B$, produce una ruptura en profundidad de las celdas de convección. La celda de convección lateral, la que está más cerca de la zona de mezcla, impulsa el flujo ascendente en el IMZ, donde se produce una mezcla con agua dulce que causa que la parte oriental del núcleo tenga densidades ligeramente menores que la zona occidental y central. Por el contrario, siguiendo un esquema de flujo clásico de frente montañoso, la zona de recarga se caracteriza principalmente por un flujo descendente de agua dulce que torna gradualmente, condicionado por la interfaz salina, hasta que finalmente asciende, con mayor rapidez, a la superficie en la MMZ. Por lo tanto, la MMZ constituye la salida principal del dominio por evaporación desde las lagunas y desde la superficie freática, que de ahí se encuentra muy próxima a la superficie del terreno.
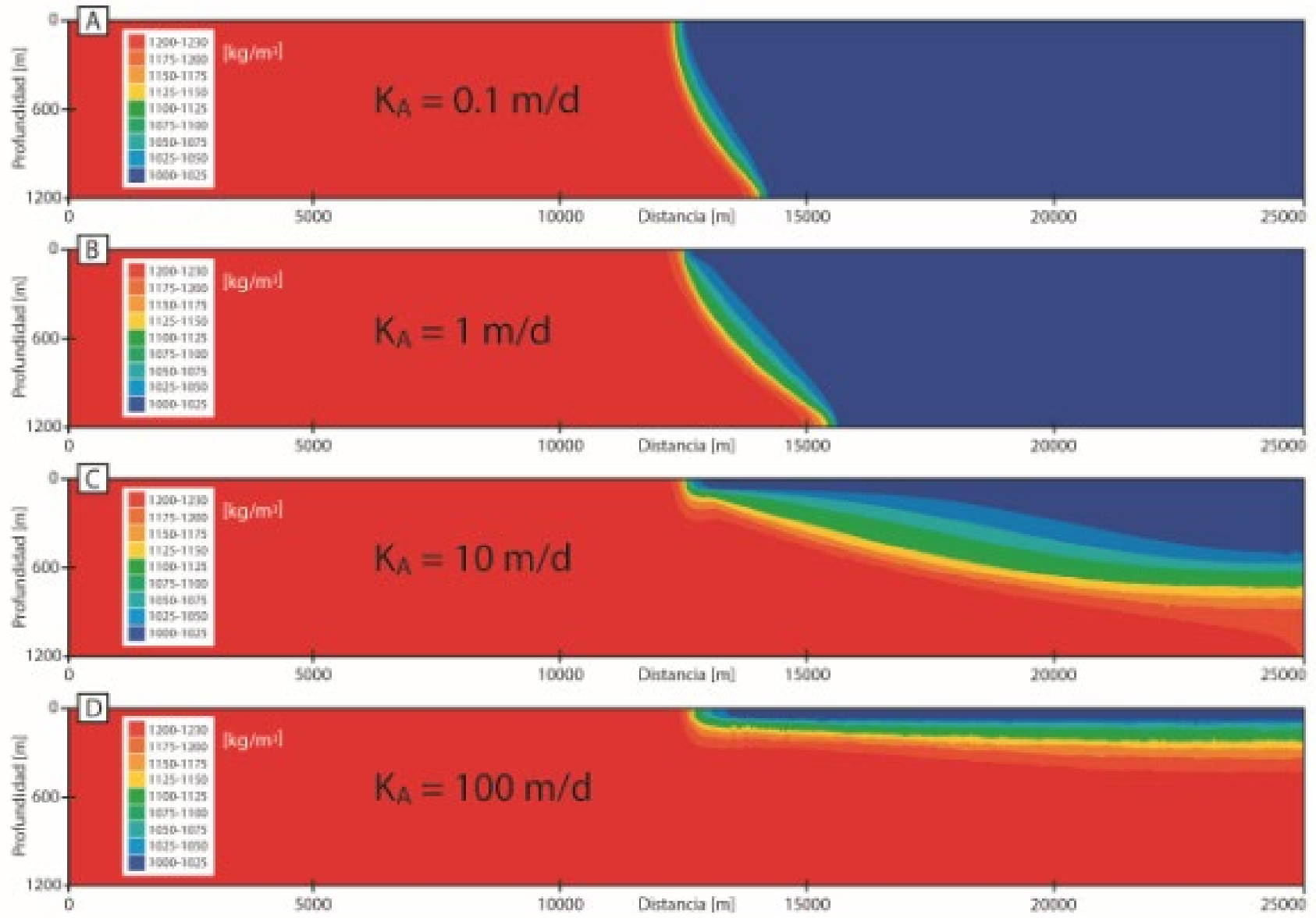

Figura 8. Resultados del análisis de sensibilidad de la conductividad hidráulica del acuífero A (Modificado de Marazuela et al., 2018).

Figure 8. Results of the sensitivity analysis of the hydraulic conductivity of aquifer $A$ (Modified from Marazuela et al., 2018). 


\section{Influencia de la heterogeneidad en la geometría de la zona de mezcla}

A través de la cartografía 3D de la zona de mezcla, se ha observado que, a escala local, su pendiente y geometría presentan irregularidades. Aunque se han llevado a cabo diferentes análisis de sensibilidad, tanto para la recarga como para la conductividad hidráulica, la conductividad hidráulica del acuífero superior (acuífero A) es la que muestra mayor sensibilidad a la geometría regional de la interfaz salina. Si se varía la recarga dentro de un rango coherente, duplicándola o reduciéndola a la mitad, los cambios son muy pequeños en comparación con los producidos por la variación de la conductividad hidráulica del acuífero $A$.

Para estudiar el efecto de una capa de alta permeabilidad en la parte superior del sistema se ha utilizado como base el modelo vertical 2D regional. Los valores de conductividad hidráulica del acuitardo $A B$ y del acuífero $B$ se fijaron ambos en $0,1 \mathrm{~m} / \mathrm{d}$ para simplificar el problema. La conductividad hidráulica del acuífero superior se modificó desde 0,1 $\mathrm{m} / \mathrm{d}$ (caso homogéneo) hasta $100 \mathrm{~m} / \mathrm{d}$. Estos valores se encuentran comúnmente en el acuífero superior de la zona de mezcla y en los abanicos aluviales del Salar de Atacama.

Los resultados del análisis de sensibilidad muestran que cuanto mayor sea la permeabilidad del acuífero superior, menor será la pendiente de la interfaz (Fig. 8). Esto ocurre porque la recarga de agua dulce flu- ye casi por completo a través de esta capa cuando la permeabilidad es muy alta. En las capas inferiores, el agua de recarga casi no penetra, por razones de potenciales hidráulicos de modo que estas quedan dentro de la zona de mezcla. En el caso contrario, cuando el acuífero es homogéneo, no hay flujo preferencial a través de la capa superior y por lo tanto el agua dulce presenta niveles piezométricos más altos y desplaza la zona de mezcla hacia el núcleo del salar, con mayor pendiente de la interfaz.

Esto explica la baja pendiente y la gran intrusión de la zona de mezcla en el Salar de Atacama, a diferencia de lo obtenido en trabajos anteriores (Tejeda et al., 2003; Vásquez et al., 2013), donde la zona de mezcla no penetraba en los abanicos aluviales y tenía una pendiente mucho más elevada al considerar medio poroso homogéneo y sin una capa superior de alta permeabilidad.

\section{Corrección de los niveles hidráulicos por variaciones de densidad}

La mejor herramienta para la gestión de los salares y sus ecosistemas es la utilización de modelos numéri$\cos 3 \mathrm{D}$ ya que permiten cuantificar y predecir el flujo subterráneo bajo diferentes escenarios. Sin embargo, la presencia de la interfaz salina condiciona la hidrodinámica de la cuenca de forma crítica por lo que es necesario incorporarla a los modelos numéricos. Esto
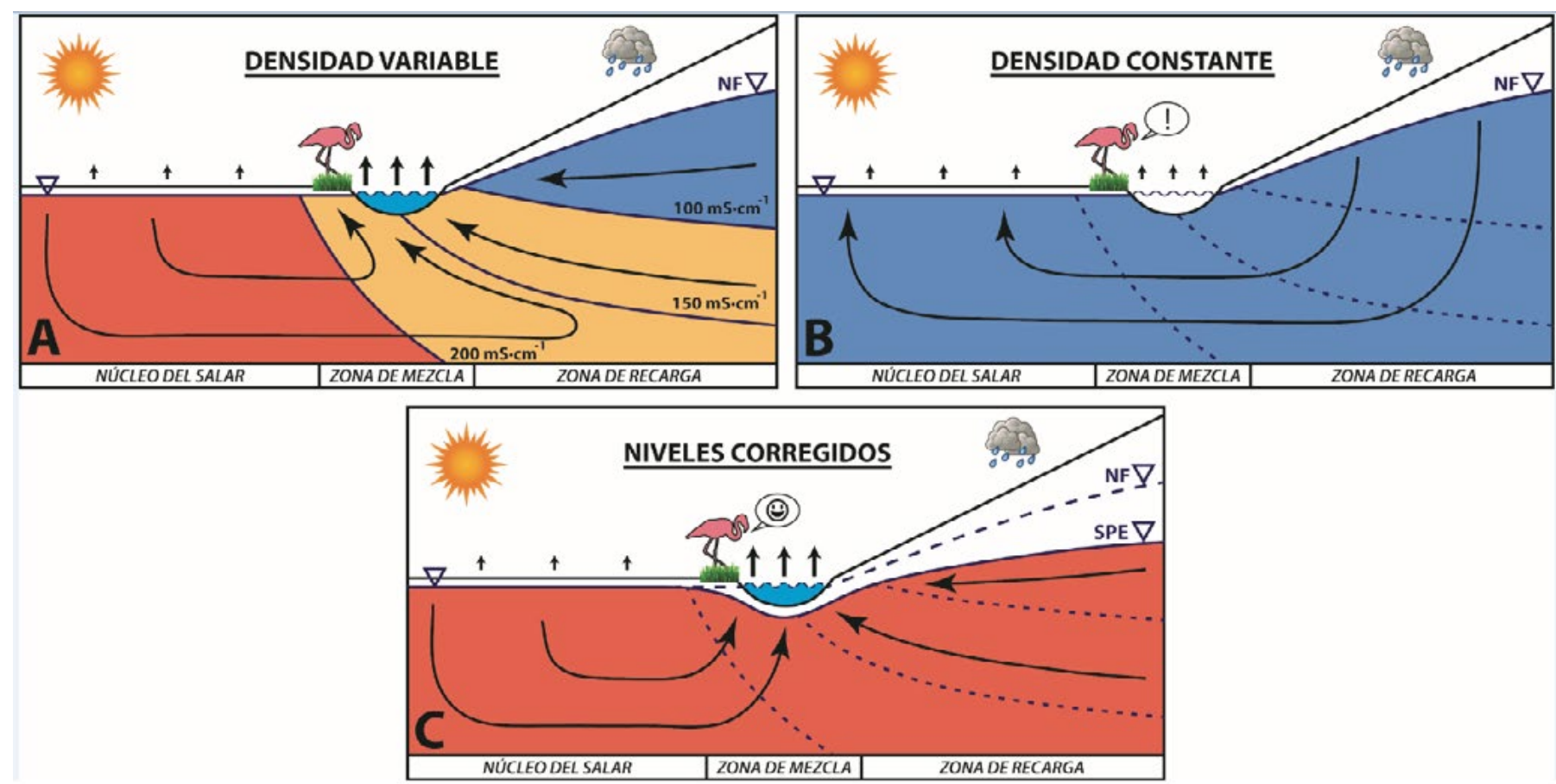

Figura 9. Dinámica de la zona de mezcla considerando diferentes escenarios. A) Flujo dominado por contrastes de densidad (situación real); B) Flujo despreciando contrastes de la densidad; C) Flujo aplicando la corrección de niveles hidráulicos. Modificado de Marazuela et al. (2018).

Figure 9. Mixing zone dynamics considering different scenarios. A) Flow dominated by density differences (present regime); B) Flow neglecting density differences; C) Flow applying water head correction. Modified from Marazuela et al. (2018). 
supone un coste computacional muy elevado y difícilmente aplicable a modelos de gestión.

En el caso de los salares, especialmente en el núcleo y zona de mezcla superficial, la componente de flujo dominante es vertical (Fig. 9A). Si se desprecia la influencia de la densidad, las líneas de flujo tendrían su origen en las montañas y su final en el núcleo (Fig. 9B). Esto contrasta con la situación real, en la que el agua subterránea descarga en la zona de mezcla superficial donde se ubican las lagunas y humedales (Fig. 9A).

Para superar la limitación del coste computacional se propone la aplicación de correcciones de densidad que permitan modelar el flujo de los salares, que está condicionado por la interfaz salina, pero con modelos de densidad constante y por lo tanto de bajo coste computacional. Una solución interesante es aplicar una metodología de corrección por densidad similar a la aplicada en los acuíferos costeros (Lusczynski, 1961) para transformar los niveles de agua salada en niveles equivalentes de agua dulce. Sin embargo, a diferencia de los acuíferos costeros, el foco de interés y el mayor número de puntos de observación en los salares se encuentra en el núcleo y en la zona de mezcla, pero no tanto en la zona de agua dulce (y área de recarga). Por esta razón, es mucho más útil desarrollar una solución que permita la corrección de los niveles piezométricos transformando los niveles de agua dulce en niveles equivalentes de salmuera.

El procedimiento detallado para la corrección de los niveles hidráulicos según las características de los piezómetros o pozos se muestra en detalle en el trabajo de Marazuela et al., (2018). Cuando los niveles de agua dulce se corrigen a niveles equivalentes de salmuera, la zona de mezcla (interfaz salina) pasa a ser el mínimo piezométrico regional y es posible reproducir los flujos verticales en la zona de mezcla superficial (Fig. 9C). Esto permite incorporar el efecto de la interfaz salina a modelos numéricos de densidad constante que sean eficientes para la gestión de los recursos hídricos por parte de la administración y de las empresas mineras. El resultado de aplicar esta metodología se muestra en el trabajo de Marazuela et al. (2019a), donde se consigue reproducir un flujo vertical en la MMZ (Fig. 10), calibrando los niveles piezométricos medidos una vez que se les ha aplicado la compensación por diferencia de densidad.

\section{Conclusiones}

Se estudió la geometría e hidrodinámica bidimensional (2D) y tridimensional (3D) de la zona de mezcla de los salares, utilizando el Salar de Atacama como caso de estudio. Esto ha permitido establecer una metodología para la gestión hidrogeológica de los recursos minerales y los valores ecológicos de los salares, que consta de tres fases: 1) muestreo de las aguas subterráneas y medición de parámetros físico-químicos, 2) cartografía 3D de la interfaz salina y 3) modelación numérica de la interfaz salina a escala local y elaboración de modelos numéricos $3 \mathrm{D}$ eficientes computacionalmente.

Esta cartografía se realizó extrapolando la información aportada por las imágenes de satélite con los da-
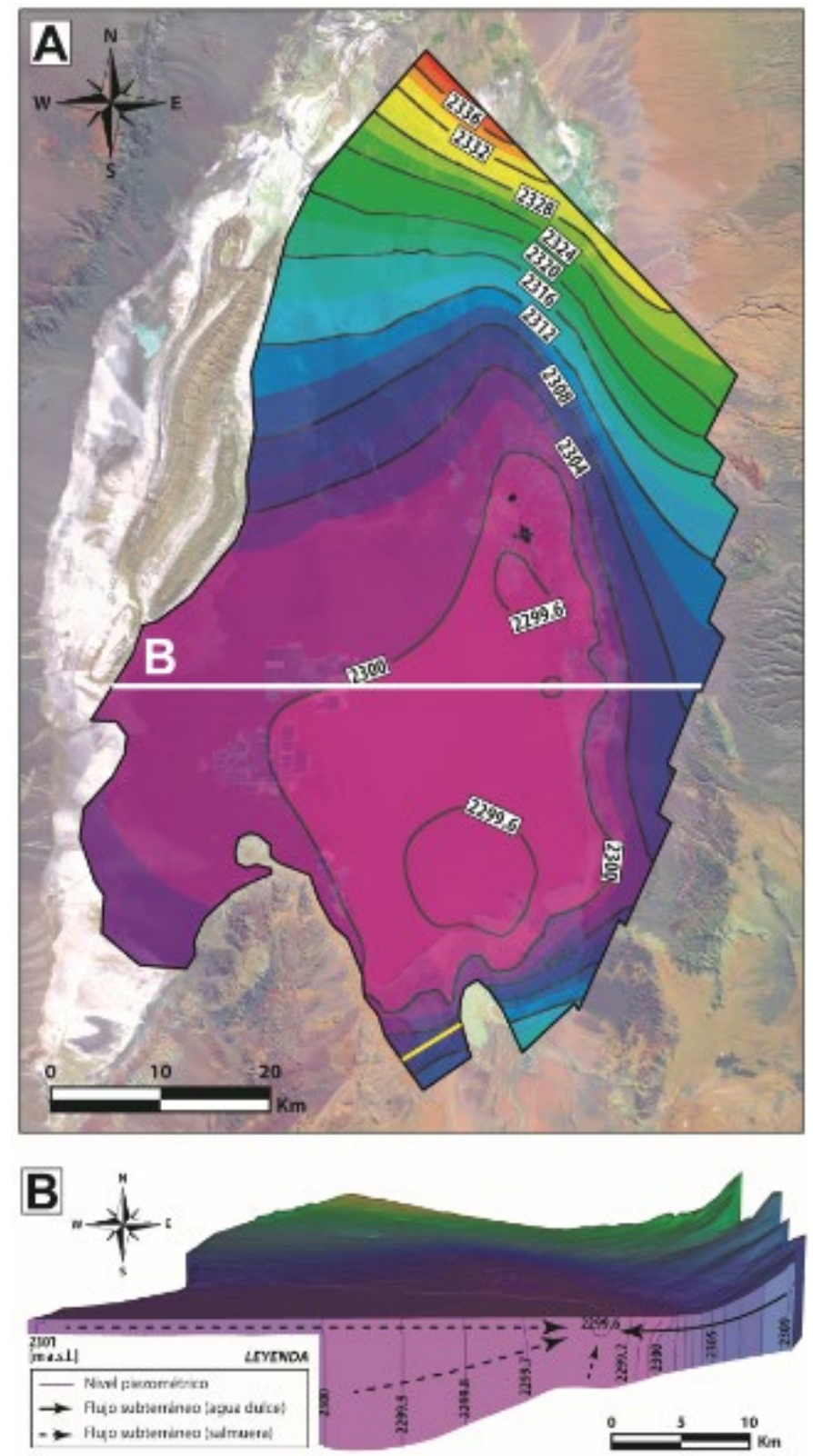

Figura 10. Resultados del modelo numérico 3D de flujo subterráneo del Salar de Atacama donde se evidencia el mínimo piezométrico regional localizado cerca de la zona de mezcla (figura A) y la correcta aproximación de los flujos verticales de la interfaz salina (figura B). Modificado de Marazuela et al. (2019a).

Figure 10. Results of the 3D groundwater flow numerical model of the Salar de Atacama, where it shows up the regional piezometric minimum, placed near the mixing zone (figure A) and the right approach taking into account the vertical flows in the saline interface (figure B). Modified from Marazuela et al. (2019a). 
tos físico-químicos medidos en los pozos de la red de monitoreo. Se evidenció una complejidad geométrica mayor y una pendiente mucho menor que la que se estimó en trabajos previos. Es la primera vez que se ha realizado una cartografía 3D de la zona de mezcla de un salar.

A través de un modelo 2D idealizado de una sección vertical de la zona de mezcla, se reprodujo la geometría de la zona de mezcla y la influencia de la heterogeneidad en la misma. Cuanto mayor la permeabilidad del acuífero superior, menor la pendiente de la zona de mezcla y mayor la intrusión de la cuña salina. Esto ocurre porque el agua dulce que se recarga fluye preferentemente a través del acuífero superior cuando la permeabilidad de la capa superior es muy alta y por lo tanto con niveles piezométricos de agua dulce menores, que permiten el desplazamiento por debajo de la cuña salina. Por lo tanto, la conductividad hidráulica del acuífero superior, generalmente constituido en los salares por evaporitas karstificadas y depósitos aluviales, es crítica para la geometría de la zona de mezcla de los salares.

Utilizando la cartografía 3D de la zona de mezcla se justifica la necesidad de aplicar una corrección de los niveles de agua dulce para compensar las variaciones de densidad y transformarlos en niveles equivalentes de salmuera. Esto reduce el coste computacional de los modelos regionales 3D de los salares cuya finalidad es la gestión de sus recursos hídricos.

\section{Agradecimientos}

Se agradece a SOM las facilidades de acceso a los datos públicos y el apoyo de sus técnicos. El trabajo se ha beneficiado de los conocimientos de la Universidad de Antofagasta a través del profesor Christian Herrera.

\section{Referencias}

Acosta, O., Custodio, E. 2008. Impactos ambientales de las extracciones de agua subterránea en el Salar del Huasco (norte de Chile). Bol. Geológico y Minero $119(1), 33-50$.

Custodio, E., 1992. Coastal aquifer salinization as a consequence of aridity: the case of Amurga phonolitic massif, Gran Canaria Island. Study and Modelling of Salt Water Intrusion. 12th SWIM-Salt Water Intrusion Meeting. CIMNE-UPC. Barcelona:81-98.

Custodio, E., Jódar, J., Herrera, C., Custodio-Ayala, J., Medina, A., 2018. Changes in groundwater reserves and radiocarbon and chloride content due to a wet period intercalated in an arid climate sequence in a large unconfined aquifer. Journal of Hydrology, 556: 427-437.

Custodio-Ayala, J, Custodio E. 2001. Hidrogeoquímica isotópica del macizo fonolítico de Amurga (SE de la Isla de Gran Canaria). En A. Medina y J. Carrera (eds.), Las Caras del Agua Subterránea. Serie Hi- drogeología y Aguas Subterráneas 1/2001. Instituto Geológico y Minero de España, Madrid, I: 461-468.

Dirección General de Aguas (DGA) 1977. Hidrogeología de la Región de Ojos de San Pedro, Proyecto CHI-535.

Duffy, C.J. y Al-Hassan, S., 1988. Groundwater circulation in a closed desert basin: topographic scaling and climatic forcing. Water Resources Research. 24 (10), 1675-1688.

Gamboa, C., Godfrey, L., Herrera, C., Custodio, E., Soler, A. 2019. The origin of solutes in groundwater in a hyper-arid environment: A chemical and multi-isotope approach in the Atacama Desert, Chile. Sci. Total Environ., 690:329-351.

GeoHidrología Consultores Ltda., 2013. Segunda Actualización del Modelo de Flujo de Agua Subterránea en el Salar de Atacama, según RCA0226/2006. Herrera C, Custodio E., 2014a. Origin of waters from small springs located at the northern coast of Chile, in the vicinity of Antofagasta. Andean Geology. 41(2): 314-341.

Herrera C, Custodio E., 2014b. Groundwater flow in a relatively old oceanic volcanic island: The Betancuria area, Fuerteventura Island, Canary Islands, Spain. Sci. Total Environ.. 496: 531-550.

Herrera, C., Custodio, E., Chong, G., Lambán, L. J., Riquelme, R., Wilke, H., Lictevout, E. 2016. Groundwater flow in a closed basin with a saline shallow lake in a volcanic area: Laguna Tuyajto, northern Chilean Altiplano of the Andes. Sci. Total Environ..,541: 303-318.

Herrera, C., Gamboa, C., Custodio, E., Jordan, T., Godfrey, L., Jódar, J., Luque, J.A., Vargas, J., Sáez, A., 2018. Groundwater origin and recharge in the hyperarid Cordillera de la Costa, Atacama Desert, northern Chile. Sci. Total Environ. 624: 114-132.

IDAEA-CSIC, 2017. Cuarta actualización del modelo hidrogeológico del Salar de Atacama. Acceso con permiso, Comisión Chilena de Energía Nuclear (CCHEN).

Jordan, T.E., Mpodozis, C., Muñoz, N., Blanco, N., Pananont, P. y Gardeweg, M., 2007. Cenozoic subsurface stratigraphy and structure of the Salar de Atacama Basin, northern Chile. Journal of South American Earth Sciences. 23, 122-146.

Lusczynski, N.J., 1961. Head and flow of ground water of variable density. Journal of Geophysical Research. 66, 4247-4256.

Marazuela, M.A., Vázquez-Suñé, E.V., Custodio, E., Palma, T., García-Gil, A. y Ayora, C., 2018. 3D mapping, hydrodynamics and modelling of the freshwater-brine mixing zone in salt flats similar to the Salar de Atacama (Chile). Journal of Hydrology,561: 223-235

Marazuela, M.A., Vázquez-Suñé, E.V., Ayora, C., García-Gil, A., Palma, T., 2019a. Hydrodynamics of salt flat basins:The Salar de Atacama example. Science of the Total Environment 651, 668-683.

Marazuela, M.A., Vázquez-Suñé, E.V., Ayora, C., Gar- 
cía-Gil, A., Palma,T., 2019b. The effect of brine pumping on the natural hydrodynamics of the Salar de Atacama:The damping capacity of salt flats. Science of the Total Environment, 654, 1118-1131.

Marazuela, M.A., Vázquez-Suñé, E., Ayora, C., García-Gil, A., 2020a. Towards more sustainable brine extraction in salt flats: Learning from the Salar de Atacama. Sci. Total Environ. 703, 135605.

Marazuela, M.A., Ayora, C., Vázquez-Suñé, E., Olivella, S., García-Gil, A., 2020b. Hydrogeological constraints for the genesis of the extreme lithium enrichment in the Salar de Atacama (NE Chile): A thermohaline flow modelling approach. Sci. Total Environ. 739, 139959.

Muñoz-Pardo, J.F., Ortiz-Astete, C.A., Mardones-Pérez, L. y Vidts-Sabelle, P., 2004. Funcionamiento hidrogeológico del acuífero del núcleo del salar de Atacama, Chile. Ingeniería Hidráulica en México. 19(3), 69-81.

Ortiz, C., Aravena, R., Briones, E., Suárez, F., Tore, C., Muñoz, J.F., 2014. Sources of surface water for the Soncor ecosystem, Salar de Atacama basin, northern Chile. Hydrological Sciences Journal. 59(2),
336-350.

Salas, J., Guimerà, J., Cornellà, O., Aravena, R., Guzmán, E., Tore, C., von Igel, W. y Moreno, R., 2010. Hidrogeología del sistema lagunar del margen este del Salar de Atacama (Chile). Boletín Geológico y Minero. 121 (4), 357-372.

Tejeda, I., Cienfuegos, R., Muñoz, J.F. y Durán, M., 2003. Numerical modeling of saline Intrusionin Salar de Atacama. Journal of Hydrologic Engineering. 8 (1), 25-34.

Universidad de Chile, 2016. Estudio estructural Zona Marginal, sector oriente del Salar de Atacama. Informe técnico para la Sociedad Química y Minera de Chile S.A. (SOM).

Urrutia,J., Herrera, C., Custodio, E., Jódar, J., Medina' A. 2019. Groundwater recharge and hydrodynamics of a complex volcanic aquifer with a shallow saline lake: Laguna Tuyajto, Andean Cordillera of northern Chile. Sci. Total Environ., (in press).

Vásquez, C., Ortiz, C., Suárez, F. y Muñoz, J.F., 2013. Modeling flow and reactive transport to explain mineral zoning in the Atacama salt flat aquifer, Chile. Journal of Hydrology. 490, 114-125. 
